\title{
Purification of Nanoparticles by Liquid Chromatography for Biomedical and Engineering Applications
}

\section{Jitendra K. Suthar, Rutuja Rokade, Ashutosh Pratinidi, Rajeshree Kambadkar, Selvan Ravindran*}

Symbiosis School of Biological Sciences, Symbiosis International University, Pune, India

Email: *selvan_ravindran@yahoo.com, *selvan.ravindran@ssbs.edu.in

How to cite this paper: Suthar, J.K., Rokade, R., Pratinidi, A., Kambadkar, R. and Ravindran, S. (2017) Purification of Nanoparticles by Liquid Chromatography for Biomedical and Engineering Applications. American Journal of Analytical Chemistry, 8, 617-624.

https://doi.org/10.4236/ajac.2017.810044

Received: September 3, 2017

Accepted: October 21, 2017

Published: October 24, 2017

Copyright $\odot 2017$ by authors and Scientific Research Publishing Inc. This work is licensed under the Creative Commons Attribution International License (CC BY 4.0).

http://creativecommons.org/licenses/by/4.0/

\begin{abstract}
Nanoparticles are extensively used for various applications in science, engineering and medicine. Synthesis of nanoparticles with high purity is essential to utilize the same in different fields of science and technology. In the present study, liquid chromatography is utilized to purify the nanoparticles. Predominantly, gold nanoparticles were synthesized from gold auric cholide and preserved in phosphate or citrate buffer. A method to purify gold nanoparticles is essential because of the possible interference from gold auric chloride and other impurities in buffer. Herein, a method has been developed using high performance liquid chromatography to purify gold nanoparticles with $100 \mathrm{~nm}$ in size from gold auric chloride and residues. UV-Vis spectroscopy was also done to ascertain the purity of the nanoparticles.
\end{abstract}

\section{Keywords}

Nanoparticles, Liquid Chromatography, Purification, UV-Vis Spectroscopy, Nanobiomaterials

\section{Introduction}

Nanoparticles are utilized for several applications in science, engineering and medicine. Nanoparticles are synthesized by chemical methods, biological methods and ball milling procedures. All these methods result in additional products as well as nanomaterials with various sizes and shapes. There are many new reports about the synthesis and characterization of nanoparticles. Silicon dioxide $\left(\mathrm{SiO}_{2}\right)$ nanoparticles composites were synthesized using ultrasonic irradiation method [1]. Poly(ortho-toluidine)-Metal (Gold and Palladium) composite na- 
nospheres were synthesized by the reaction between $\mathrm{Au}$ or Pd colloidal solution with o-toluidine [2]. Graphene quantum dots were prepared by combining ultrasonic method along with chemical activation by potassium hydroxide [3]. Titanium dioxide nanofibres in the size range of $30-50 \mathrm{~nm}$ in diameter were developed by electrospinning process and structural changes were performed using organo-silane agents by a coupling reaction and grafted with carbohydrate molecules [4]. Silver nanoparticles coated with Titanium dioxide nanofiber composites were developed by a novel chemical method. Titanium dioxide nanofibres were dispersed in silver salt solution by ultrasonification, followed by addition of reducing agent to prepare composite [5]. Thus many methods developed for synthesis of nanoparticles involve chemicals or biologicals as starting materials. Therefore purification of nanoparticle is mandatory to utilize them for various applications. In the present study, liquid chromatography is demonstrated as a tool to purify the nanoparticles. Monolayer protected gold nanoclusters were characterized using high performance liquid chromatography [6] in one of the reports. Otherwise, studies were not performed routinely using liquid chromatography to characterize nanoparticles.

Understanding and dealing with size range of $1-100$ nanometers is termed as nanotechnology [7]. Gold and Silver nanoparticles have been in use for a long time for various applications such as painting and decorative coloring. Nanoparticles were also used for engineering applications and biomedical applications [8] [9]. Gold and Silver nanoparticles also possess antibacterial properties and medical applications [10]. Gold and Silver are inert metals and therefore, suitable for biological and medical applications [10] [11] [12]. Properties of a metal are altered drastically from bulk to micro and nanometer size range [13] [14] [15]. Gold and silver are metals and therefore not soluble in solvents, whereas nanoparticles are extensively soluble in solvents. Hence, size of the particle plays an important role to decide the function and applications of nanomaterials. Applications of nanomaterials are due to its chemical and physical properties. Optical properties of various sizes of nanoparticles are due to its absorption at definite wavelength of light resulting in particular color. Gold is a yellowish metal, but gold nanoparticles have colors ranging from light pink to dark red depending on its size. This optical property of nanoparticles was exploited by UV-Visible spectroscopy to confirm a nanoparticle corresponding to a particular size [16]. Transmission electron microscope is used to understand the size of the particles, infrared spectroscopy and mass spectrometry to observe the molecular changes and powder x-ray diffraction to know the size and crystalline nature of the samples [17] [18] [19].

In the present study, liquid chromatography with photo diode array detector is employed to identify the nanoparticle and also to purify the nanoparticle. Synthesis or biosynthesis of nanoparticles as well as coated nanoparticles could consist of pure nanoparticles, biosurfactant stabilized nanoparticles and biosurfactants, therefore chromatography is essential to purify the nanoparticles [20]. 
Column chromatographies with single quardupole mass spectrometers are extensively used in the field of pharmaceutical chemistry to characterize pharmaceutical drugs and metabolites [21] [22]. Similarly liquid chromatography hyphenated with tandem mass spectrometry was utilized to characterize pharmaceutical drugs and its metabolites [23] [24] [25]. Pesticides in food industry, pollutants in environment and many medical applications were achieved utilizing liquid chromatography and mass spectrometry. Nanomaterials were also used in pharmaceutical industry for drug discovery and development [26]. Therefore, purification and characterization of nanomaterials by simple technique is essential.

\section{Materials and Methods}

\subsection{Materials}

Gold nanoparticle, $100 \mathrm{~nm}$ in size (CAS no: 742031) and gold auric chloride (CAS no: 27988-77-8) were purchased from Sigma Aldrich, St. Louis, MO, USA. Solvents Acetonitrile and Methanol were purchased from Sigma Aldrich, St. Louis, MO, USA. Deionized water is produced using Millipore system, Merck, USA. Thermo hypersil gold C18 column was purchased from Thermo Fischer Scientific, USA.

\subsection{Chromatography Conditions}

The HPLC system used for the study was Shimadzu Prominence liquid chromatography (Shimadzu, Kyoto, Japan) equipped with LC-20 AD pump, SIL-20 AC injector valve with sample holder, C10-10AS column holder with oven, DGu-20 AS degasser, LC-20 AD pump and SPD M20A photo diode array detector. The HPLC column used was C18 column with $150 \mathrm{~mm} \times 5 \mathrm{~mm}$ i.d, consisting of 5 micron particles of C18 packing material. The quantification of peaks was performed using LC-Quant software associated with the Shimadzu HPLC instrument. Gradient mobile phase conditions were used for acetonitrile/water; methanol/water and acetonitrile/water systems. Appropriate solvent system was chosen based on the gradient method. From the gradient method Acetonitrile/Water system with 70:30 ratio was identified as the ideal mobile phase condition to analyze the sample. Acetonitrile/Water system with 70:30 ratio was employed to identify gold auric chloride and gold nanoparticles. Ten microliter of the sample was injected for the analysis and oven temperature of the column was maintained at 40 degrees.

\subsection{UV-Vis Spectroscopy}

Ultraviolet-Visible (UV-Vis) spectroscopy is performed for the procured gold nanoparticles using Thermo UV-Vis instrument. Gold nanoparticles of $100 \mathrm{~nm}$ size were dispersed in citrate buffer. Samples were analyzed directly and the wavelength assigned for $100 \mathrm{~nm}$ gold nanoparticles dispersed in buffer specified by Sigma Aldrich was observed. Quality of the purchased gold nanoparticle was 
confirmed based on UV-Vis spectroscopic studies.

\section{Results and Discussion}

UV-Vis spectroscopy is performed for the procured gold nanoparticles with 100 $\mathrm{nm}$ size. Gold nanoparticles were observed at a wavelength of $564 \mathrm{~nm}$ as shown in Figure 1. Gold nanoparticles were reddish pink in colour and observed wavelength as recommended by the vendor Sigma Aldrich signifies the stability of the gold nanoparticles. Gold nanoparticles exhibit a characteristic optical property as surface plasmon resonance that arises due to the interaction of incident light with a specific wavelength upon the collective oscillation of electrons in the conduction band of gold nanoparticles. Size of the nanaoparticle and wavelength of incident light has direct correlation [27]. It has been confirmed that UV-Vis band shifts to higher wavelength as the size of the nanoparticle increases. In the present study, the observed band around $560 \mathrm{~nm}$ corresponds to $100 \mathrm{~nm}$ particle size. The observed band around $560 \mathrm{~nm}$ could be due to the aggregation of gold nanoparticles or aggregation of citrate molecules over the gold nanoparticles. One of the recent reports suggests janus structure that exist in gold nanoparticles could influence the aggregation behavior of nanoparticles [28].

Liquid chromatography is demonstrated as an important tool to purify and characterize gold nanoparticles. Gold nanoparticles and gold nanoparticles coated with various biological samples were synthesized from gold auric chloride as the starting material. Gold nanoparticles with various ligands are generally obtained using gold auric chloride and ligand in the presence of reducing agents. Hence, Gold auric chloride could be a possible contaminant in the gold nanoparticle samples. Liquid chromatography method has been developed for both gold auric chloride and gold nanoparticle. Gold auric chloride elutes at the retention time of $2 \mathrm{~min}$ and gold nanoparticle elutes at the retention time of 2.7 min as shown in Figure 2 and Figure 3.

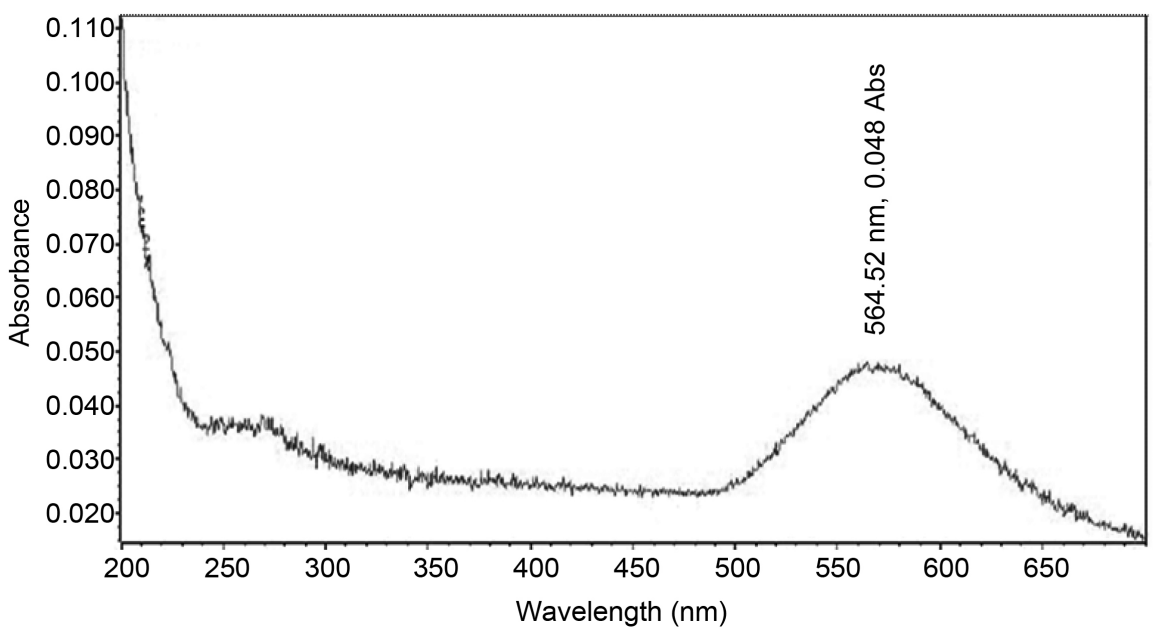

Figure 1. UV-Vis Spectrum of $100 \mathrm{~nm}$ Gold nanoparticle dispersed in buffer. Broad band observed at $564 \mathrm{~nm}$ is characteristic of $100 \mathrm{~nm}$ gold nanoparticle. 


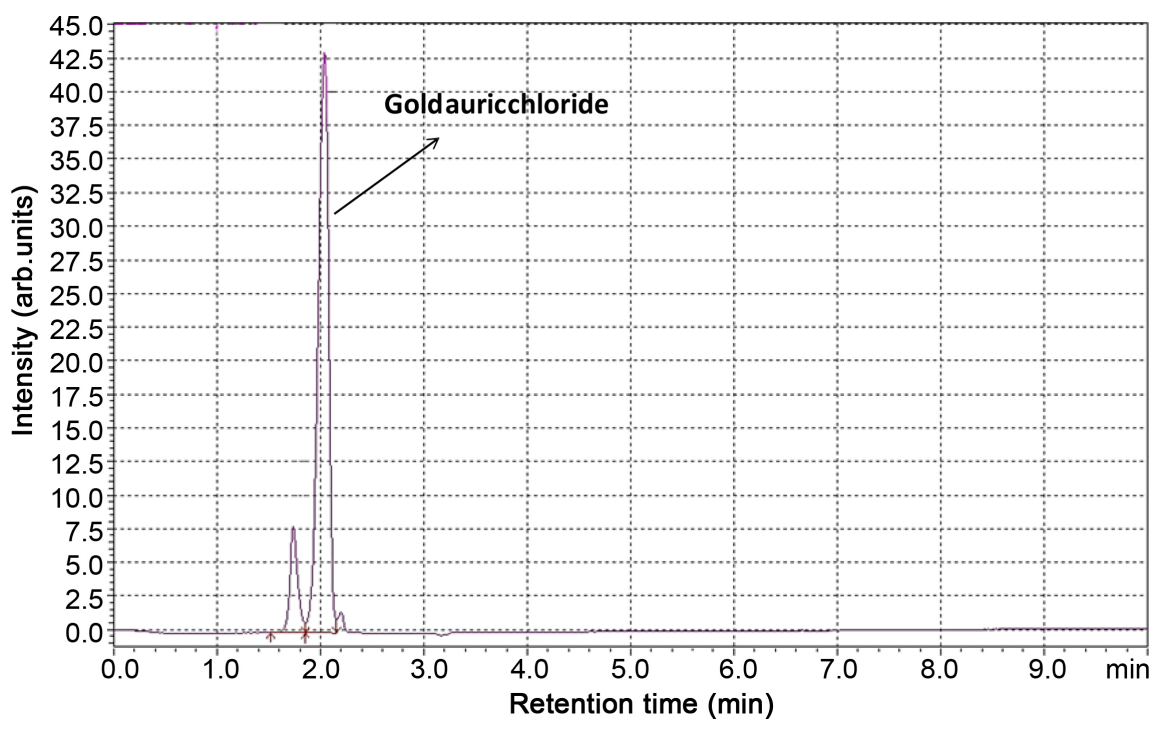

Figure 2. High performance liquid chromatography of gold auric chloride. Gold auric chloride (labeled) elutes at the retention time of $2 \mathrm{~min}$. Co eluting minor peak at $1.8 \mathrm{~min}$ could be an impurity from buffer.

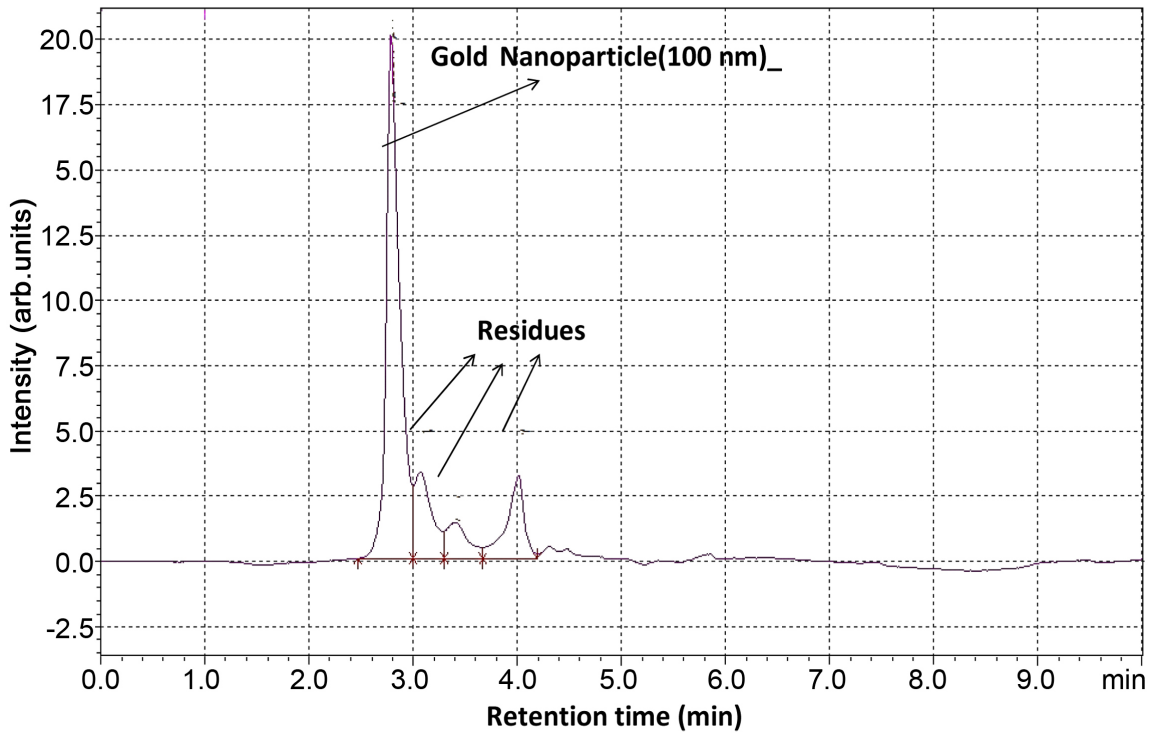

Figure 3. High performance liquid chromatography of $100 \mathrm{~nm}$ gold nanoparticle. Gold nanoparticle with $100 \mathrm{~nm}$ in size elutes at the retention time of $2.8 \mathrm{~min}$. Peaks eluting between retention time of 3 to 4 min could be due to residues in the sample.

Additional peaks observed in the nanoparticle sample could be due to the citrate buffer used to disperse the nanoparticle. Both gold auric chloride and gold nanoparticle with size range of $100 \mathrm{~nm}$ elutes at different retention time and hence, it suggests that high performance liquid chromatography can be used to purify the gold nanoparticles and scale up the nanoparticles in high quantity. Method developed in the current analytical HPLC, if replicated in preparative HPLC gold nanoparticles can be purified and obtained in high quantity.

Most of the previous reports have utilized only UV-Vis spectroscopy to con- 
firm the nanoparticle based on wavelength. The present study using liquid chromatography confirms the utility of HPLC as both purification and characterization tool for nanoparticles. In one of the previous study [20], monolayer protected gold clusters were studied using liquid chromatography. Gold nanoparticles protected with hexane thiolate ligands were separated from mixed monolayers of hexanethiolate and mercaptoundecanoic acid using reverse phase $\mathrm{C} 8$ column under the mobile phase condition of 6:1 acetone/toluene at a flow rate of $1 \mathrm{ml} / \mathrm{min}$ [20]. In this reported study, gold clusters were separated based on its size and small clusters eluted first followed by medium sized and large clusters. Elution of gold clusters also depended on stationary phase and mobile phase used.

Similarly in the present study, interaction between gold nanaoparticles of 100 $\mathrm{nm}$ size and gold auric chloride with stationary phase (i.e. C18 column) and mobile phase (water and acetonitrile) decides the retention time and purification. Gold auric chloride is more polar and elutes faster at retention time $2 \mathrm{~min}$ (Figure 2) than that of gold nanoparticle in the size range of $100 \mathrm{~nm}$ that elutes at the retention time of $2.8 \mathrm{~min}$ (Figure 3). This difference in retention time between gold auric chloride and gold nanoparticle is significant enough to purify the nanoparticles from the starting material. Intensity of the gold auric chloride and gold nanoparticle confirms the recovery of the sample and hence the developed method could be utilized to quantify the nanoparticles and gold chloride with precision. Thus liquid chromatography proves to be an important technology to purify and characterize nanoparticles.

\section{Conclusion and Future Perspectives}

Method has been developed to purify and characterize nanoparticles using liquid chromatography. Standard nanoparticle of size $100 \mathrm{~nm}$ was used to test the method. Same method is applicable for gold auric chloride that is generally used to synthesize gold nanoparticles. Gold nanoparticle and gold auric chloride elute at different retention times, thus the method is suitable to purify the nanoparticle from starting material and also from impurities in the buffers to preserve the nanomaterials. Transferring the method from current analytical HPLC to preparative HPLC could help in scaling up the product in high yield with great purity. Apart from this application in the present study, liquid chromatography can also be utilized to purify nanomaterials obtained from silver, copper, zinc, titanium, iron and alloys. Liquid chromatography interfaced with mass spectrometry can be utilized to identify and quantify the mass of the purified nanomaterials and also the possible structure. Thus purified nanomaterials derived from various metals will be highly beneficial for biomedical scientists and engineers to develop products of high quality that are safe to be utilized in many applications.

\section{Acknowledgements}

Authors thank Symbiosis International University for funding and facility to 
carry out the research. Authors are thankful to Dr. Vinaykumar Rale and Dr. Anurdha Vaidya for insightful discussions.

\section{Conflict of Interest}

No Conflict of interest.

\section{References}

[1] Zhang, Y.P., Lee, S.H., Reddy, K.R., Gopalan, A.I. and Lee, K.P. (2007) Synthesis and Characterization of Core-Shell $\mathrm{SiO}_{2}$ Nanoparticles/Poly(3-aminophenylboronic acid Complexes. Journal of Applied Polymer Science, 104, 2743-2750.

https://doi.org/10.1002/app.25938

[2] Reddy, K.R., Lee, K.P. and Gopalan, A.I. (2007) Self-Assembly Directed Synthesis of Poly(ortho-toluidine)-Metal (Gold and Palladium) Composite Nanospheres. Journal of Nanoscience and Nanotechnology, 9, 3117-3125. https://doi.org/10.1166/jnn.2007.692

[3] Hassan, M., Haque, E., Reddy, K.R., Minett, A.I., Chen, J. and Gomes, V.J. (2014) Edge-Enriched Graphene Quantum Dots for Enhanced Photoluminescence and Supercapacitance. Nanoscale, 6, 11988-11994. https://doi.org/10.1039/C4NR02365J

[4] Reddy, K.K., Gomes, V.G. and Hassan, M. (2014) Carbon Functionalized $\mathrm{TiO}_{2} \mathrm{Na-}$ nofibres for High Efficiency Photocatalysis. Materials Research Express, 1, Article ID: 015012. https://doi.org/10.1088/2053-1591/1/1/015012

[5] Reddy, K.R., Nakata, K.O., Tsuyoshi, M.T., Tryk, D.A. and Fujishima, A. (2011) Facile Fabrication and Photocatalytic Application of Ag Nanoparticles- $\mathrm{TiO}_{2}$ Nanofiber Composites. Journal of Nanoscience and Nanotechnology, 11, 3692-3695. https://doi.org/10.1166/jnn.2011.3805

[6] Jimenez, V.L., Leopold, M.C., Mazitelli, C., Jorgenson, J.W. and Murray, R.W. (2003) HPLC of Monolayer-Protected Gold Nanoclusters. Analytical Chemistry, 75, 199-206. https://doi.org/10.1021/ac0260589

[7] Feynman, R. (1991) There's a Plenty of Room at the Bottom. Science, 254, 1300-1301.

[8] Guo, D., Xie, G. and Luo, J. (2013) Mechanical Properties of Nanoparticles: Basics and Applications. Journal of Physics D: Applied Physics, 47, 1-25.

[9] Salata, O.V. (2004) Applications of Nanoparticles in Biology and Medicine. Journal of Nano Biotechnology, 2, 1-6. https://doi.org/10.1186/1477-3155-2-3

[10] Dykman, L.A. and Khlebtsov. (2011) Gold Nanoparticles in Biology and Medicine: Recent Advances and Prospects. Acta Naturae, 3, 34-55.

[11] Samuel, U. and Guggenbichler, J.P. (2004) Prevention of Catheter Related Infections: The Potential of a New Nano-Silver Impregnated Catheter. International Journal of Antimicrobial Agents, 23, S75-S78.

[12] Eckhardt, S., Brunetto, P.S., Gagnon, J., Priebem, B., Geese, B. and Fromm, K.M. (2004) Nanobio Silver: Its Interactions with Peptides and Bacteria, and Its Uses in Medicine. Chemical Reviews, 113, 4708-4754. https://doi.org/10.1021/cr300288v

[13] Mody, V.V., Swale, R., Singh, A. and Mody, H.R. (2010) Introduction to Metallic Nanoparticles. Journal of Pharmacy and Bioallied Sciences, 2, 282-289. https://doi.org/10.4103/0975-7406.72127

[14] Kelly, K.L., Coronado, E., Zhao, L.L. and Schatz, G.C. (2003) The Optical Properties of Metal Nanoparticles: The Influence of Size, Shape and Dielectric Environment. 
The Journal of Physical Chemistry B, 107, 668-667. https://doi.org/10.1021/jp026731y

[15] Tajiri, T., Saisho, S., Mito, M., Deguchi, H., Konishi, K. and Kohno, A. (2015) Size Dependence of Crystal Structure and Magnetic Properties of NiO Nanoparticles in Mesoporous Silica. The Journal of Physical Chemistry C, 119, 1194-1200.

[16] Amendola, V. and Meneghetti, M. (2009) Size Evaluation of Gold Nanoparticles by UV-vis Spectroscopy. The Journal of Physical Chemistry C, 113, 4277-4285. https://doi.org/10.1021/jp8082425

[17] Selvan, R. and Pradeep, T. (1999) Towards the Synthesis and Characterization of Metallocarbohedrenes. Chemical Physics Letters, 309, 149-156.

[18] Selvan, R., Unnikrishnan, T., Ganapathy, S. and Pradeep, T. (2000) Macroscopic Synthesis and Characterization of Giant Fullerenes. Chemical Physics Letters, 316, 205-210.

[19] Showkat, A.M., Zhang, Y.P., kim, M.S., Gopalan, A.I., Reddy, K.K and Lee, K.P. (2007) Analysis of Heavy Metal Toxic Ions by Adsoprtion on to Amino-Functionalized Ordered Mesoporous Silica. Bulletin of the Korean Chemical Society, 28, 1985-1992. https://doi.org/10.5012/bkcs.2007.28.11.1985

[20] Singh, P., Ravindran, S., Suthar, J.K., Deshpande, P., Rokade, R. and Rale, V. (2017) Production of Biosurfactant Stabilized Nanoparticles. International Journal of Pharma and Bio Sciences, 8, 701-707. https://doi.org/10.22376/ijpbs.2017.8.2.b701-707

[21] Ravindran, S., Zharikova, O.L., Hill, R.A., Nanovskaya, T.N., Hankins, G.D.V. and Ahmed, M.S. (2006) Identification of Glyburide Metabolites Formed by Hepatic and Placental Microsomes of Humans and Baboons. Biochemical Pharmacology, 72, 1730-1737.

[22] Zharikova, O.L., Ravindran, S., Nanovskaya, T.N., Hankins, G.D.V. and Ahmed, M.S. (2007) Kinetics of Glybride Metabolism by Hepatic and Placental Microsomes. Biochemical Pharmacology, 73, 2012-2019.

[23] Ravindran, S., Basu, S., Gorti, S., Surve, P. and Sloka, N. (2013) Metabolic Profile of Glyburide in Human Liver Microsomes using LC-DAD-Q-TRAP-MS/MS. Biomedical Chromatography, 27, 575-582. https://doi.org/10.1002/bmc.2830

[24] Ravindran, S., Jadhav, A., Surve, P., Lonsane, G., Honrao, P. and Nanda, B. (2014) Technologies and Strategies to Characterize and Quantitate Metabolites in Drug Discovery and Development. Biomedical Chromatography, 28, 1547-1553. https://doi.org/10.1002/bmc.3309

[25] Ravindran, S., Gokhale, D., Suthar, J.K., Rokade, R., Deshpande, P. and Singh, P. (2017) ADME of Glyburide, Metformin and Nutrition for Management of Gestational Diabetes. Journal of Endocrine Disorders, 4, 1-4.

[26] Subrahmanyam, S., Ahmed, T., Pinjari, J., Patole, P., Ravindran, S., Gangal, R., Wangikar, P., Basu, S. and Rastogi, H. (2012) Translational Drug Discovery Research: Integration of Medicinal Chemistry, Computational Modeling, Pharmacology, ADME and Toxicology. Encyclopedia of Drug Metabolism and Interactions, Chapter 18, 1-54.

[27] Haiss, A., Thank, N.T.K., Aveyard, J. and Fernig, D.G. (2007) Determination of Size and Concentration of Gold Nanoparticles from UV-Vis Spectra. Analytical Chemistry, 79, 4215-4221. https://doi.org/10.1021/ac0702084

[28] Rercebom, A.M., Casares, J.J.C., Claes, N., Bals, S., Loh, A. and Marzan L.M.L. (2016) Janus Gold Nanoparticles Obtained via Spontaneous Binary Polymer Shell Segregation. Chemical Communications, 52, 4278-4281. https://doi.org/10.1039/C5CC10454H 